\title{
Association between dietary calcium intake and arterial stiffness according to dietary vitamin $D$ intake in men
}

\author{
Hirokazu Uemura $^{1 *}$, Sakurako Katsuura-Kamano ${ }^{1}$, Miwa Yamaguchi ${ }^{1}$, Mariko Nakamoto ${ }^{2}$, \\ Mineyoshi Hiyoshi ${ }^{1}$ and Kokichi Arisawa ${ }^{1}$ \\ ${ }^{1}$ Department of Preventive Medicine, Institute of Health Biosciences, The University of Tokushima Graduate School, \\ 3-18-15 Kuramoto-cho, Tokushima 770-8503, Japan \\ ${ }^{2}$ Department of Public Health and Applied Nutrition, Institute of Health Biosciences, The University of Tokushima \\ Graduate School, 3-18-15 Kuramoto-cho, Tokushima 770-8503, Japan
}

(Submitted 27 January 2014 - Final revision received 30 June 2014 - Accepted 3 July 2014 - First published online 5 September 2014)

\section{Abstract}

Studies on the associations of dietary $\mathrm{Ca}$ and vitamin $\mathrm{D}$ intakes with arterial stiffness are scarce. In the present study, these associations were evaluated in Japanese men. Data from a total of 535 eligible men, aged 35-69 years, who participated in the baseline survey of a cohort study in Tokushima Prefecture, Japan, and underwent brachial-ankle pulse wave velocity (ba-PWV) measurements were analysed. ba-PWV is a measure of arterial stiffness and is recognised as a marker of atherosclerotic vascular damage. Information regarding the cohort's lifestyle characteristics including dietary behaviour over the past year was obtained from a structured self-administered questionnaire. Dietary Ca and vitamin D intakes were adjusted for total energy intake using the residual method and divided into quartiles; the highest quartile was used as the reference. General linear models were used to evaluate the associations between dietary Ca and vitamin D intakes and ba-PWV values adjusted for probable covariates. The association between dietary Ca intake and ba-PWV was further evaluated using similar general linear models stratified by dietary vitamin D intake (median or below/above median). Dietary Ca intake was found to be significantly inversely associated with ba-PWV after adjusting for probable covariates $(P$ for trend=0.020). However, no such association was observed between dietary vitamin D intake and ba-PWV. The inverse association between dietary Ca intake and ba-PWV was striking in subjects with higher dietary vitamin D intake. However, no association was found in subjects with lower dietary vitamin $\mathrm{D}$ intake. These results indicate that adequate dietary $\mathrm{Ca}$ and vitamin $\mathrm{D}$ intakes may be protective against the development of arterial stiffness in Japanese men.

\section{Key words: Arterial stiffness: Calcium: Vitamin D: Cross-sectional studies}

Cardiovascular health is influenced by various factors including ageing, sex, genetic factors and lifestyle factors including dietary behaviours. Besides its critical role in skeletal metabolism, the potential effects of $\mathrm{Ca}$ on non-skeletal health outcomes, such as CVD, have recently drawn attention ${ }^{(1)}$. Some epidemiological studies have reported inverse associations of $\mathrm{Ca}$ intake with cardiovascular risk and mortality ${ }^{(2,3)}$. Similarly, vitamin D deficiency has been suggested to be associated with various health disorders including $\mathrm{CVD}^{(4)}$. In contrast, excessive $\mathrm{Ca}$ intake via supplementation has recently been suggested to adversely affect vascular events ${ }^{(5)}$.

Atherosclerotic changes in arteries mainly contribute to the pathogenesis of CVD, and increased arterial stiffness is associated with atherosclerosis. Arterial stiffness can be assessed by measuring pulse wave velocity (PWV); carotid-femoral
PWV is an established index for assessing aortic stiffness ${ }^{(6)}$ However, measuring carotid-femoral PWV is rather complicated and time consuming. Alternatively, brachial-ankle pulse wave velocity (ba-PWV) measurement is convenient, reproducible and relatively quick. Although ba-PWV reflects the arterial stiffness of both large- and mid-sized arteries ${ }^{(7,8)}$, it is well correlated with carotid-femoral PWV as well as aortic PWV assessed using a direct catheter method ${ }^{(8)}$. Therefore, ba-PWV measurement has become widespread for screening arterial atherosclerotic changes in Asian countries. Despite epidemiological findings of inverse associations of Ca intake with the risk factors for CVD, little is known about the associations of dietary $\mathrm{Ca}$ and vitamin $\mathrm{D}$ intakes with arterial stiffness. In the present study, the associations of dietary $\mathrm{Ca}$ and vitamin $\mathrm{D}$ intakes with arterial stiffness on

Abbreviations: ABI, ankle-brachial pressure index; ba-PWV, brachial-ankle pulse wave velocity; PWV, pulse wave velocity.

*Corresponding author: Dr H. Uemura, fax +81 886337074 , email uemura@basic.med.tokushima-u.ac.jp 
the basis of ba-PWV were evaluated in Japanese men, whose mean daily $\mathrm{Ca}$ intake is considerably lower than that of Westerners. Because dietary $\mathrm{Ca}$ and vitamin $\mathrm{D}$ may act synergistically, their combined associations with measures of arterial stiffness were also evaluated.

\section{Methods}

\section{Study subjects}

A total of 574 men aged 35-69 years who participated in the baseline survey of a prospective cohort study in Tokushima Prefecture, Japan, from November 2009 to January 2012 and underwent ba-PWV measurements were included in the present cross-sectional study. The subjects were mostly office workers and not shift workers. The present study was carried out as part of the Japan Multi-Institutional Collaborative Cohort (J-MICC) Study, a prospective cohort study ${ }^{(9)}$. Briefly, the J-MICC Study aims to examine the associations of lifestyle and genetic factors, as well as their interactions, with lifestyle-related diseases. All participants of the J-MICC Study provided written informed consent before participation. The present study was conducted according to the guidelines laid down in the Declaration of Helsinki, and the ethics committees of Nagoya University School of Medicine (with which the former principal investigator, Nobuyuki Hamajima, is affiliated), Aichi Cancer Center (with which the current principal investigator, Hideo Tanaka, is affiliated) and the University of Tokushima Graduate School approved the study protocol.

\section{Questionnaire}

Information regarding individual lifestyle characteristics over the past year was obtained through a structured selfadministered questionnaire. All the responses were checked by trained staff at the time of the survey. Leisure-time exercise was estimated on the basis of the International Physical Activity Questionnaire ${ }^{(10)}$. Exercise was divided into three levels: light exercise (e.g. walking and hiking); moderate exercise (e.g. light jogging and swimming); vigorous exercise (e.g. marathon running and competitive sports). The degrees of leisure-time exercise for the three levels were expressed as metabolic equivalent (MET)-h/week (MET level $\times$ hours of activity $\times$ events per week) and summed. In this estimation, light, moderate and vigorous exercise levels were assigned to be $3 \cdot 4,7 \cdot 0$ and $10 \cdot 0 \mathrm{MET}$, respectively.

Dietary intake was evaluated using a validated short FFQ in the baseline survey of the J-MICC Study ${ }^{(11-14)}$. This FFQ included questions about the dietary intake of forty-seven varieties of foods and beverages (i.e. green tea, coffee and alcohol) over the previous year. Information regarding the intake frequency and amounts of the three staple foods (i.e. rice, bread and noodles) consumed at breakfast, lunch and dinner was obtained. The intake volume and frequency of alcoholic beverages including sake, beer, Shōchū (a Japanese distilled beverage), Chūhai (a sweetened beverage mixed with Shōchū), whisky and wine were determined. Only intake frequency was determined for the other forty-three foods and beverages. Daily total energy $(\mathrm{kJ} / \mathrm{d})$, $\mathrm{Ca}(\mathrm{mg} / \mathrm{d})$ and vitamin $\mathrm{D}(\mu \mathrm{g} / \mathrm{d})$ intakes were calculated using a program developed by the Department of Public Health, Nagoya City University School of Medicine ${ }^{(11,12)}$.

\section{Pulse wave velocity measurement}

ba-PWV was measured using a waveform analyser (Model BP203RPE III; Colin Company Limited) as described previously ${ }^{(7)}$. Briefly, the subjects were examined while resting in the supine position in an air-conditioned room. Extremity blood pressure was measured using an oscillometric method, and the anklebrachial pressure index (ABI) was automatically calculated. ba-PWV was calculated using time-phase analysis between the right brachial artery pressure and volume waveforms at both ankles. PWV is a measure of the speed of the pulse wave travelling over a specific distance in the artery. This speed of the pulse wave increases as the stiffness of artery wall increases; therefore, a higher PWV value indicates increased arterial stiffness. To reduce inter-observer variation, all ba-PWV measurements were performed by a single researcher throughout the study. Individual ba-PWV and ABI data are expressed as the means of the bilateral ba-PWV and ABI, respectively.

\section{Anthropometric and biochemical measurements}

Body height was obtained from the questionnaire, while body weight was measured to the nearest $0 \cdot 1 \mathrm{~kg}$ during the baseline survey. BMI was calculated as weight (kg) divided by height $(\mathrm{m})$. Venous blood was drawn from each subject, and serum was separated within $3 \mathrm{~h}$. Serum lipid concentrations were measured at an external laboratory (BML, Inc.). Total cholesterol and TAG concentrations were determined using an enzyme assay, and HDL-cholesterol concentrations were determined using a direct method.

\section{Statistical analyses}

Among the 574 men initially included in the present study, nineteen with a history of IHD or stroke as well as five who had a low right or left ABI $(\leq 0 \cdot 9)$, indicating peripheral arterial occlusive disease, were excluded. Apart from them, five subjects whose daily total energy intake was extremely high $(>16800 \mathrm{~kJ} / \mathrm{d}$; this is almost equal to $>4000 \mathrm{kcal} / \mathrm{d})$ or low $(<4200 \mathrm{~kJ} / \mathrm{d}$; this is equal to $<1000 \mathrm{kcal} / \mathrm{d})$ or who were taking $\mathrm{Ca}$ or vitamin $\mathrm{D}$ supplements were excluded. After excluding an additional ten subjects for whom serum lipid concentration data were missing, a total of 535 men were included in the analyses.

Dietary Ca and vitamin D intakes were adjusted for total energy intake after log transformation using the residual method and divided into quartiles in the analyses such that the number of subjects in each category was nearly equal; the highest quartile, which was thought to represent relatively sufficient intake, was used as the reference.

Continuous variables are expressed as means and standard deviations or medians with their 25 th and 75 th percentiles. Categorical variables are expressed as the proportion (\%). ANOVA, 
the Kruskal-Wallis test and Fisher's exact test were used to compare the baseline characteristics among the quartile categories of dietary Ca or vitamin D intake where appropriate. General linear models were used to evaluate the associations of dietary $\mathrm{Ca}$ and vitamin $\mathrm{D}$ intakes with ba-PWV after adjusting for the following probable covariates: age (continuous) and systolic blood pressure $(<120,120-<130,130-<140,140-<160$, or $\geq 160 \mathrm{mmHg}$ with no medical treatment or antihypertensive agent use), which are recognised as being very closely associated with arterial stiffness, in model 1 and age, systolic blood pressure, BMI ( $\mathrm{kg} / \mathrm{m}^{2}$, quartiles), current smoking status (no/yes), current drinking status (no/yes), leisure-time exercise (MET-h/week, quartiles), hypercholesterolaemia ( $\geq 5.7 \mathrm{mmol} / 1$ or $\geq 220 \mathrm{mg} / \mathrm{dl}$ or receiving medical treatment, no/yes), low HDL-cholesterol concentrations $(<1.0 \mathrm{mmol} / \mathrm{l}$ or $<40 \mathrm{mg} / \mathrm{dl}, \mathrm{no} / \mathrm{yes})$, elevated TAG concentrations ( $\geq 1.7 \mathrm{mmol} / \mathrm{l}$ or $\geq 150 \mathrm{mg} / \mathrm{dl}$, no/yes), diabetes (receiving medical treatment, no/yes), and daily energy intake (continuous, log-transformed) in model 2. Tests for trends were performed by assigning the ordinal categorical variables of 1,2 , 3 and 4 for each quartile of dietary $\mathrm{Ca}$ and vitamin $\mathrm{D}$ intakes. The association between dietary $\mathrm{Ca}$ intake and ba-PWV was further evaluated using similar general linear models stratified by dietary vitamin $\mathrm{D}$ intake (median or below/above median) to assess whether dietary vitamin $\mathrm{D}$ intake had a modifying effect on the association between dietary Ca intake and arterial stiffness. Interaction terms of two exposure variables (Ca intake, continuous, log-transformed; vitamin D intake, dichotomous) were created and included in the model to assess statistical interactions.

All calculations and statistical tests were performed using SAS (version 8.2; SAS Institute, Inc.). All statistical tests were based on two-sided probabilities, and the level of significance was set at $P<0.05$.

\section{Results}

The mean age and BMI of the study subjects were 50.0 (SD 8.6) years and $24.4(\mathrm{sD} 3.3) \mathrm{kg} / \mathrm{m}^{2}$, respectively. The mean ba-PWV value was $1436(\mathrm{SD} 237) \mathrm{cm} / \mathrm{s}$.

\section{Baseline characteristics of the subjects according to dietary calcium and vitamin D intakes}

The baseline characteristics of the subjects are summarised in Tables 1 and 2 according to their dietary $\mathrm{Ca}$ and vitamin D intakes, respectively. Subjects with lower dietary Ca intake were younger, had a higher current smoking status and had a lower level of leisure-time exercise. Subjects with the lowest dietary $\mathrm{Ca}$ intake had highest systolic blood pressure and highest ba-PWV. Subjects with lower dietary vitamin D intake were younger and had higher values of low HDL-cholesterol concentrations. ba-PWV was not statistically different among the vitamin D intake quartiles.

\section{Associations of dietary calcium and vitamin D intakes with arterial stiffness}

The associations of dietary Ca and vitamin D intakes with ba-PWV in the general linear models are summarised in Table 3.
Lower dietary Ca intake was significantly associated with increased ba-PWV after adjusting for the multivariable covariates ( $P$ for trend $=0.020$ in model 2 ). No adjusted associations were found between dietary vitamin $\mathrm{D}$ intake and ba-PWV.

\section{Association between dietary calcium intake and arterial stiffness stratified according to dietary vitamin D intake}

Stratified analyses revealed a strikingly significant inverse association between dietary $\mathrm{Ca}$ intake and ba-PWV in subjects whose dietary vitamin $\mathrm{D}$ intake was greater than the median $(P$ for trend $=0.019$ in model 2 ; Table 4 ). In contrast, no such association was observed in subjects whose dietary vitamin D intake was less than or equal to the median ( $P$ for trend $=0.761$ in model 2 ; Table 4 ). The interactions of dietary Ca intake (continuous, log-transformed) and vitamin D intake (dichotomous) with ba-PWV were marginally significant even after adjusting for the multivariable covariates ( $P$ for interaction $=0.058$ in model 2).

\section{Discussion}

In the present study, the inverse association between dietary Ca intake and arterial stiffness was investigated after adjusting for the classical atherosclerotic risk factors in Japanese men.

$\mathrm{Ca}$ is the most abundant mineral in the human body. Besides its core role in skeletal metabolism, $\mathrm{Ca}$ is required for numerous critical biological functions including muscle contraction, vascular tone and various enzyme-mediated processes ${ }^{(15)}$. Some prospective studies have reported inverse associations between $\mathrm{Ca}$ intake and the risk of stroke incidence ${ }^{(2)}$ and cardiovascular mortality ${ }^{(3)}$. In the present study, insufficient dietary Ca intake was found to be associated with increased ba-PWV in Japanese men. Because humans cannot produce $\mathrm{Ca}$, sufficient $\mathrm{Ca}$ intake is required for Ca homeostasis. However, the mean daily $\mathrm{Ca}$ intake of the Japanese population remains below the recommendation levels ${ }^{(16)}$ (650 and $700 \mathrm{mg} / \mathrm{d}$ for men aged $30-49$ and $\geq 50$ years, respectively; 650 and $600 \mathrm{mg} / \mathrm{d}$ for women aged $30-69$ years and $\geq 70$ years, respectively) $)^{(17)}$ and is far lower than that of Westerners ${ }^{(18)}$.

Studies on the associations between dietary Ca intake and arterial stiffness are scarce. Kesse-Guyot et al. ${ }^{(19)}$ have reported that a nutritionally poor dietary pattern including low $\mathrm{Ca}$ intake is related to increased stiffening of large arteries, which is consistent with the present findings. There are several possible explanations for the inverse association between $\mathrm{Ca}$ intake and arterial stiffness. The leading explanation is the hypotensive effect of $\mathrm{Ca}$. Dietary $\mathrm{Ca}$ intake or supplementation was found to be inversely associated with systolic blood pressure in a cross-sectional study in Japan ${ }^{(20)}$ and in some meta-analyses of randomised controlled trials ${ }^{(21,22)}$. In the present study, subjects with the lowest dietary Ca intake had highest systolic blood pressure; this finding is in agreement with those of other studies ${ }^{(20-22)}$. Experimental studies suggest that $\mathrm{Ca}$ is involved in the regulation of vascular smooth muscle cell contractility ${ }^{(23,24)}$ and the down-regulation of the reninangiotensin system ${ }^{(25,26)}$; this system contributes to proper blood pressure regulation. Ca has also been demonstrated to 
Table 1. Baseline characteristics of the subjects according to dietary calcium intake*

(Mean values and standard deviations; median values with their 25th and 75th percentiles; number of subjects and percentages)

\begin{tabular}{|c|c|c|c|c|c|c|c|c|c|}
\hline & \multicolumn{8}{|c|}{ Ca intake $(\mathrm{mg} / \mathrm{d})$} & \multirow[b]{3}{*}{$P$} \\
\hline & \multicolumn{2}{|c|}{$\begin{array}{c}\mathrm{Q1} \\
(\leq 351 \cdot 8)\end{array}$} & \multicolumn{2}{|c|}{$\begin{array}{c}\text { Q2 } \\
(>351 \cdot 8-412 \cdot 2)\end{array}$} & \multicolumn{2}{|c|}{$\begin{array}{c}\text { Q3 } \\
(>412 \cdot 2-497 \cdot 3)\end{array}$} & \multicolumn{2}{|c|}{$\begin{array}{c}\text { Q4 } \\
(>497 \cdot 3)\end{array}$} & \\
\hline & Mean & SD & Mean & SD & Mean & SD & Mean & SD & \\
\hline$n$ & \multicolumn{2}{|c|}{134} & \multicolumn{2}{|c|}{134} & \multicolumn{2}{|c|}{134} & \multicolumn{2}{|c|}{133} & \\
\hline$\%$ & \multicolumn{2}{|c|}{$25 \cdot 0$} & \multicolumn{2}{|c|}{$25 \cdot 0$} & \multicolumn{2}{|c|}{$25 \cdot 0$} & \multicolumn{2}{|c|}{24.9} & \\
\hline Age (years) & $47 \cdot 6$ & $8 \cdot 2$ & $48 \cdot 7$ & $8 \cdot 3$ & $48 \cdot 7$ & $8 \cdot 9$ & $50 \cdot 9$ & $8 \cdot 6$ & 0.016 \\
\hline BMI $\left(\mathrm{kg} / \mathrm{m}^{2}\right)$ & & & & & & & & & 0.936 \\
\hline Median & \multicolumn{2}{|c|}{$24 \cdot 1$} & \multicolumn{2}{|c|}{$24 \cdot 1$} & \multicolumn{2}{|c|}{23.9} & & & \\
\hline 25th and 75th percentiles & $22 \cdot$ & & 22 . & & $22 \cdot$ & & 22. & & \\
\hline Current smoking status & & & & & & & & & 0.001 \\
\hline No & & & & & & & & & \\
\hline$n$ & & & & & & & & & \\
\hline$\%$ & & & & & & & & & \\
\hline Yes & & & & & & & & & \\
\hline$n$ & & & & & & & & & \\
\hline$\%$ & & & & & & & & & \\
\hline Current drinking status & & & & & & & & & 0.574 \\
\hline No & & & & & & & & & \\
\hline$n$ & & & & & & & & & \\
\hline$\%$ & & & & & & & & & \\
\hline Yes & & & & & & & & & \\
\hline$n$ & & & & & & & & & \\
\hline$\%$ & & & & & & & & & \\
\hline Exercise (MET-h/week) & & & & & & & & & 0.013 \\
\hline Median & & & & & & & & & \\
\hline 25th and 75 th percentiles & 0.43 & & $1 \cdot 28$ & & 1.28 & & $1 \cdot 28$ & & \\
\hline Systolic BP $(\mathrm{mmHg})$ & $136 \cdot 9$ & $16 \cdot 0$ & $135 \cdot 1$ & $20 \cdot 0$ & $131 \cdot 2$ & $15 \cdot 4$ & $134 \cdot 4$ & $15 \cdot 5$ & 0.044 \\
\hline Diastolic BP (mmHg) & 85.6 & $10 \cdot 9$ & 84.6 & $13 \cdot 1$ & $82 \cdot 0$ & $11 \cdot 1$ & 84.3 & $10 \cdot 7$ & 0.066 \\
\hline Energy intake $(\mathrm{kJ} / \mathrm{d})$ & & & & & & & & & 0.050 \\
\hline Median & & & & & & & & & \\
\hline 25th and 75 th percentiles & 677 & & 665 & 13 & 700 & & 699 & 339 & \\
\hline Total cholesterol $(\mathrm{mmol} / \mathrm{l})$ & 5.44 & 0.93 & 5.46 & 0.93 & 5.44 & 0.79 & $5 \cdot 56$ & 0.78 & 0.555 \\
\hline HDL-cholesterol (mmol/l) & 1.45 & 0.29 & 1.45 & 0.30 & 1.46 & 0.31 & 1.46 & 0.33 & 0.973 \\
\hline TAG $(\mathrm{mmol} / \mathrm{l})$ & & & & & & & & & 0.628 \\
\hline Median & & & & & & & & & \\
\hline 25th and 75 th percentiles & 0.8 & & 0.8 & & 0.9 & & 0.9 & & \\
\hline Prevalence & & & & & & & & & \\
\hline Hypertension & & & & & & & & & 0.485 \\
\hline$n$ & & & & & & & & & \\
\hline$\%$ & & & & & & & & & \\
\hline Hypercholesterolaemia & & & & & & & & & 0.313 \\
\hline$n$ & & & & & & & & & \\
\hline$\%$ & & & & & & & & & \\
\hline Low HDL-cholesterol concentrations & & & & & & & & & 0.991 \\
\hline$n$ & & & & & & & & & \\
\hline$\%$ & & & & & & & & & \\
\hline Elevated TAG concentrations & & & & & & & & & 0.732 \\
\hline$n$ & & & & & & & & & \\
\hline$\%$ & & & & & & & & & \\
\hline Diabetes & & & & & & & & & $0 \cdot 155$ \\
\hline$n$ & & & & & & & & & \\
\hline$\%$ & & & & & & & & & \\
\hline $\mathrm{ABI}$ & $1 \cdot 11$ & 0.06 & $1 \cdot 12$ & 0.06 & $1 \cdot 12$ & 0.06 & $1 \cdot 13$ & 0.06 & 0.139 \\
\hline ba-PWV (cm/s) & 1480 & 264 & 1431 & 207 & 1400 & 227 & 1434 & 242 & 0.048 \\
\hline
\end{tabular}

$\mathrm{Q}$, quartiles; MET, metabolic equivalents; $\mathrm{BP}$, blood pressure; $\mathrm{ABI}$, ankle-brachial pressure index; ba-PWV, brachial-ankle pulse wave velocity.

${ }^{*} \mathrm{Ca}$ intake was adjusted for total energy intake after log transformation using the residual method.

be involved in the reduction of platelet aggregation ${ }^{(27)}$ and regulation of lipoprotein metabolism ${ }^{(28,29)}$. Dietary Ca promotes the formation of insoluble complexes with fatty acids, reducing fatty acid absorption ${ }^{(29)}$. Moreover, Ca increases lipid excretion by binding to bile acids; dietary $\mathrm{Ca}$ subsequently lowers LDL-cholesterol synthesis ${ }^{(30)}$. In the present study, serum LDL-cholesterol concentrations were found to be not associated with dietary $\mathrm{Ca}$ intake; this might be due to the low Ca intake in the study subjects. Further studies are required to elucidate the effect of $\mathrm{Ca}$ intake on circulating lipid profiles.

Excessive $\mathrm{Ca}$ intake via supplementation has recently been suggested to adversely affect vascular events ${ }^{(5)}$. Taking $\mathrm{Ca}$ or 
Table 2. Baseline characteristics of the subjects according to dietary vitamin $D$ intake*

(Mean values and standard deviations; median values with their 25th and 75th percentiles; number of subjects and percentages)

\begin{tabular}{|c|c|c|c|c|c|}
\hline & \multicolumn{4}{|c|}{ Vitamin D intake $(\mu \mathrm{g} / \mathrm{d})$} & \multirow[b]{3}{*}{$P$} \\
\hline & Q1 ( $\leq 4.42)$ & $\mathrm{Q} 2(>4.42-5.08)$ & Q3 $(>5.08-7.35)$ & Q4 (>7.35) & \\
\hline & Mean & Mean & Mean & Mean & \\
\hline$n$ & 134 & 134 & 134 & 133 & \\
\hline$\%$ & $25 \cdot 0$ & $25 \cdot 0$ & $25 \cdot 0$ & 24.9 & \\
\hline Age (years) & $46 \cdot 6$ & $49 \cdot 0$ & $48 \cdot 4$ & $52 \cdot 0$ & $<0.001$ \\
\hline $\mathrm{BMI}\left(\mathrm{kg} / \mathrm{m}^{2}\right)$ & & & & & 0.782 \\
\hline Median & $23 \cdot 6$ & 23.9 & $24 \cdot 1$ & 24.5 & \\
\hline 25th and 75th percentiles & $22 \cdot 1,25 \cdot 9$ & $21 \cdot 9,26 \cdot 3$ & $22 \cdot 6,25 \cdot 5$ & $22 \cdot 2,26 \cdot 0$ & \\
\hline Current smoking status & & & & & 0.252 \\
\hline No & & & & & \\
\hline$n$ & 80 & 88 & 89 & 95 & \\
\hline$\%$ & $59 \cdot 7$ & $65 \cdot 7$ & $66 \cdot 4$ & 71.4 & \\
\hline Yes & & & & & \\
\hline$n$ & 54 & 46 & 45 & 38 & \\
\hline$\%$ & $40 \cdot 3$ & $34 \cdot 3$ & $33 \cdot 6$ & $28 \cdot 6$ & \\
\hline Current drinking status & & & & & 0.553 \\
\hline No & & & & & \\
\hline$n$ & 35 & 39 & 37 & 29 & \\
\hline$\%$ & $26 \cdot 1$ & $29 \cdot 1$ & $27 \cdot 6$ & $21 \cdot 8$ & \\
\hline Yes & & & & & \\
\hline$n$ & 99 & 95 & 97 & 104 & \\
\hline$\%$ & $73 \cdot 9$ & $70 \cdot 9$ & $72 \cdot 4$ & $78 \cdot 2$ & \\
\hline Exercise (MET-h/week) & & & & & 0.052 \\
\hline Median & 2.55 & $5 \cdot 25$ & $5 \cdot 10$ & $5 \cdot 10$ & \\
\hline 25th and 75th percentiles & $0.00,12.99$ & $1 \cdot 28,15 \cdot 30$ & $1 \cdot 28,12 \cdot 33$ & $1 \cdot 28,15 \cdot 30$ & \\
\hline Systolic BP (mmHg) & $134 \cdot 2 \quad 16 \cdot 6$ & $131.9 \quad 16 \cdot 3$ & $\begin{array}{ll}134.5 & 17 \cdot 7\end{array}$ & $137 \cdot 0 \quad 16 \cdot 8$ & 0.114 \\
\hline Diastolic BP (mmHg) & 83.9 & 83.2 & 83.8 & 85.6 & 0.334 \\
\hline Energy intake $(\mathrm{kJ} / \mathrm{d})$ & & & & & $<0.001$ \\
\hline Median & 7699 & 7481 & 7155 & 7761 & \\
\hline 25th and 75th percentiles & 7025,9025 & 6908,8033 & 6351,7958 & 7058,8347 & \\
\hline Total cholesterol (mmol/l) & $\begin{array}{ll}5.55 & 0.88\end{array}$ & $\begin{array}{ll}5.45 & 0.80\end{array}$ & $5.45 \quad 0.96$ & $5.55 \quad 0.79$ & 0.633 \\
\hline HDL-cholesterol (mmol/l) & 1.43 & 1.44 & 1.46 & 1.48 & 0.463 \\
\hline TAG $(\mathrm{mmol} / \mathrm{l})$ & & & & & 0.666 \\
\hline Median & 1.36 & 1.28 & $1 \cdot 20$ & $1 \cdot 30$ & \\
\hline 25th and 75th percentiles & $0.95,1.94$ & $0.86,2.02$ & $0.87,1.74$ & $0.89,1.81$ & \\
\hline Prevalence & & & & & \\
\hline Hypertension & & & & & 0.160 \\
\hline$n$ & 57 & 57 & 58 & 72 & \\
\hline$\%$ & $42 \cdot 5$ & $42 \cdot 5$ & $43 \cdot 3$ & $54 \cdot 1$ & \\
\hline Hypercholesterolaemia & & & & & 0.957 \\
\hline$n$ & 52 & 53 & 56 & 55 & \\
\hline$\%$ & $38 \cdot 8$ & $39 \cdot 6$ & $41 \cdot 8$ & $41 \cdot 4$ & \\
\hline Low HDL-cholesterol concentrations & & & & & 0.050 \\
\hline$n$ & 11 & 4 & 7 & 2 & \\
\hline$\%$ & $8 \cdot 2$ & $3 \cdot 0$ & $5 \cdot 2$ & 1.5 & \\
\hline Elevated TAG concentrations & & & & & 0.795 \\
\hline$n$ & 45 & 42 & 38 & 39 & \\
\hline$\%$ & $33 \cdot 6$ & $31 \cdot 3$ & 28.4 & $29 \cdot 3$ & \\
\hline Diabetes & & & & & 0.093 \\
\hline$n$ & 2 & 4 & 8 & 9 & \\
\hline$\%$ & 1.5 & $3 \cdot 0$ & $6 \cdot 0$ & $6 \cdot 8$ & \\
\hline $\mathrm{ABI}$ & 0.07 & 0.06 & 0.06 & $1 \cdot 12$ & 0.920 \\
\hline ba-PWV (cm/s) & 1435 & 1411 & 1419 & 1480 & 0.085 \\
\hline
\end{tabular}

$\mathrm{Q}$, quartiles; MET, metabolic equivalents, $\mathrm{BP}$, blood pressure; $\mathrm{ABI}$, ankle-brachial pressure index; ba-PWV, brachial-ankle pulse wave velocity.

* Vitamin $D$ intake was adjusted for total energy intake after log-transformation using the residual method.

vitamin D supplements is not popular among Japanese people, especially men. Furthermore, subjects taking Ca or vitamin D supplements were excluded from the analyses carried out in the present study. Therefore, it is thought that none of the subjects was consuming excessive Ca or vitamin D.

Vitamin D is a fat-soluble vitamin obtained from foods, beverages and supplements and is also generated by cutaneous synthesis resulting from sun exposure. Vitamin D insufficiency has recently drawn attention as a potential risk factor for CVD. Studies on the association between dietary vitamin D intake and arterial stiffness are scarce and their results are inconsistent $^{(31,32)}$. In the present study, no multivariateadjusted association was found between dietary vitamin D intake and arterial stiffness. One possible reason for the lack 
Table 3. Associations of dietary calcium and vitamin $D$ intakes with brachial-ankle pulse wave velocity $(\mathrm{cm} / \mathrm{s})$ (Adjusted mean values with their standard errors)*

\begin{tabular}{|c|c|c|c|c|c|c|}
\hline & \multicolumn{3}{|c|}{ Model 1† } & \multicolumn{3}{|c|}{ Model $2 \ddagger$} \\
\hline & Adjusted mean & SE & $P$ & Adjusted mean & SE & $P$ \\
\hline \multicolumn{7}{|l|}{ Ca intake $(\mathrm{mg} / \mathrm{d})$} \\
\hline Q1 $(\leq 351 \cdot 8)$ & 1521 & 15 & 0.003 & 1561 & 29 & 0.010 \\
\hline Q2 (>351.8-412.2) & 1468 & 15 & 0.620 & 1507 & 27 & 0.927 \\
\hline Q3 (>412.2-497.3) & 1466 & 16 & 0.694 & 1512 & 28 & 0.738 \\
\hline Q4 (>497.3) & 1457 & 16 & Reference & 1505 & 28 & Reference \\
\hline$P$ for trend & & 0.004 & & & 0.020 & \\
\hline \multicolumn{7}{|l|}{ Vitamin D intake $(\mu \mathrm{g} / \mathrm{d})$} \\
\hline Q1 $(\leq 351.8)$ & 1496 & 15 & 0.240 & 1533 & 28 & 0.448 \\
\hline Q2 (>351.8-412.2) & 1470 & 16 & 0.964 & 1509 & 29 & 0.770 \\
\hline Q3 (>412.2-497.3) & 1473 & 16 & 0.915 & 1509 & 28 & 0.760 \\
\hline Q4 (>497.3) & 1471 & 16 & Reference & 1515 & 29 & Reference \\
\hline$P$ for trend & & 0.283 & & & 0.484 & \\
\hline
\end{tabular}

Q, quartiles.

${ }^{*} \mathrm{Ca}$ and vitamin $\mathrm{D}$ intakes were adjusted for total energy intake after log transformation using the residual method.

† Model 1: adjusted for age and systolic blood pressure.

$\ddagger$ Model 2: adjusted for age, systolic blood pressure, BMI, current smoking status, current drinking status, leisure-time exercise, hyper-

cholesterolaemia, low HDL-cholesterol concentrations, elevated TAG concentrations, diabetes and daily energy intake.

of such an association is that vitamin D intake from the diet is lower than that from supplements. Therefore, the body burden level of vitamin $\mathrm{D}$ did not reach the effective level. Another possible serious reason is that we did not have information about cutaneous vitamin D synthesis from sun exposure or data on circulating 25-hydroxyvitamin D concentration, which is an index of systemic vitamin D status, as we focused on the association between dietary factors (foods and beverages) and arterial stiffness. The systemic body burden level of vitamin D cannot be explained by dietary vitamin D intake alone. A recent study ${ }^{(33)}$ in Korean adolescents has demonstrated that seasonal differences in the amount of sunlight exposure affect serum 25-hydroxyvitamin D concentrations and furthermore mean serum 25-hydroxyvitamin D concentrations over the year are associated with the intake frequencies of vitamin $\mathrm{D}$ food sources. We evaluated the mean intake frequencies of foods and beverages over the previous year and therefore our estimate of dietary vitamin $\mathrm{D}$ intake may be associated to some extent with mean serum 25-hydroxyvitamin D concentrations over the year. Otherwise, there may in fact be no association between vitamin D intake and arterial stiffness. Thus, further studies including circulating 25-hydroxyvitamin D measurement are required to determine the association between systemic vitamin D status and arterial stiffness.

Interestingly, in the present study, a strong inverse association was found between dietary $\mathrm{Ca}$ intake and arterial stiffness in subjects with higher dietary vitamin D intake. However, no association was found in subjects with lower dietary vitamin D intake. Among subjects with higher dietary vitamin D intake, an elevation

Table 4. Association between dietary calcium intake and brachial-ankle pulse wave velocity (cm/s) stratified by dietary vitamin $\mathrm{D}$ intake

(Adjusted mean values with their standard errors)

\begin{tabular}{|c|c|c|c|c|c|c|c|c|}
\hline & \multicolumn{4}{|c|}{ Model 1‡ } & \multicolumn{4}{|c|}{ Model $2 \S$} \\
\hline & Adjusted mean & SE & $P$ & $P$ for interaction* & Adjusted mean & SE & $P$ & $P$ for interaction* \\
\hline \multicolumn{9}{|c|}{ Lower vitamin D intake ( $\leq$ median) } \\
\hline \multicolumn{9}{|c|}{ Ca intake $(\mathrm{mg} / \mathrm{d})$} \\
\hline Q1 $(\leq 351.8)$ & 1469 & 16 & 0.541 & & 1476 & 38 & 0.645 & \\
\hline Q2 (>351.8-412.2) & 1443 & 19 & 0.780 & & 1448 & 39 & 0.628 & \\
\hline Q3 (>412.2-497.3) & 1469 & 21 & 0.589 & & 1475 & 39 & 0.706 & \\
\hline Q4 (>497.3) & 1452 & 24 & Reference & & 1463 & 42 & Reference & \\
\hline$P$ for trend & \multicolumn{3}{|c|}{0.689} & \multirow{2}{*}{\multicolumn{4}{|c|}{0.761}} & \\
\hline & & & & & & & & 0.058 \\
\hline \multicolumn{9}{|c|}{ Higher vitamin D intake (>median) } \\
\hline \multicolumn{9}{|c|}{ Ca intake $(\mathrm{mg} / \mathrm{d})$} \\
\hline Q1 $(\leq 351 \cdot 8)$ & 1628 & 33 & $<0.001$ & & 1675 & 51 & $<0.001$ & \\
\hline Q2 (>351.8-412.2) & 1488 & 22 & 0.689 & & 1541 & 44 & 0.943 & \\
\hline Q3 $(>412 \cdot 2-497 \cdot 3)$ & 1470 & 23 & 0.812 & & 1534 & 47 & 0.876 & \\
\hline Q4 (>497.3) & 1477 & 20 & Reference & & 1539 & 44 & Reference & \\
\hline$P$ for trend & \multicolumn{3}{|c|}{0.002} & \multicolumn{5}{|c|}{0.019} \\
\hline
\end{tabular}

Q, quartiles.

${ }^{*} P$ value for interaction of $\mathrm{Ca}$ intake (continuous, log-transformed) and vitamin $\mathrm{D}$ intake (dichotomous, $\leq$ median and $>$ median).

$\dagger \mathrm{Ca}$ and vitamin $\mathrm{D}$ intakes were adjusted for total energy intake after log transformation using the residual method.

$\ddagger$ Model 1: adjusted for age and systolic blood pressure.

$\S$ Model 2: adjusted for age, systolic blood pressure, BMI, current smoking status, current drinking status, leisure-time exercise, hypercholesterolaemia, low HDL-cholesterol concentrations, elevated TAG concentrations, diabetes and daily energy intake. 
of about $140 \mathrm{~cm} / \mathrm{s}$ in multivariate-adjusted ba-PWV values was observed in the lowest quartile of $\mathrm{Ca}$ intake compared with the highest quartile. In a recent study carried out in Japan, it has been found that an elevation of $100 \mathrm{~cm} / \mathrm{s}$ in ba-PWV indicates a significant increase in age- and sex-adjusted hazard ratio for future vascular events (hazard ratio 1.06, 95\% CI 1.01, 1.10; $P=0.015$ per $100 \mathrm{~cm} / \mathrm{s}$ elevation in ba-PWV) ${ }^{(34)}$. Therefore, the difference of $140 \mathrm{~cm} / \mathrm{s}$ in ba-PWV is considered to have a considerable impact on future vascular events. The human body has difficulty in absorbing Ca. Activated vitamin D enhances the intestinal absorption of $\mathrm{Ca}^{(15)}$. Therefore, $\mathrm{Ca}$ absorption is more efficient in individuals with higher dietary vitamin D intake, and protective effects of $\mathrm{Ca}$ (and vitamin D) against hypertension and atherosclerosis including direct action on the vascular system might be greater in individuals with higher dietary vitamin D intake. However, whether dietary vitamin D supplementation contributes to the reduction of ba-PWV cannot be discussed based on only our data. Dietary supplementation of both $\mathrm{Ca}$ and vitamin $\mathrm{D}$ is probably effective at reducing ba-PWV.

The present study has several limitations. First, because of the cross-sectional study design, the causal relationship between $\mathrm{Ca}$ intake and arterial stiffness should be interpreted with caution. Second, information about dietary and other lifestyle factors was self-reported; therefore, non-differential misclassification may have been inevitable. Similarly, Ca and vitamin D intakes were estimated on the basis of self-reported information from a short FFQ, and their estimations were relatively low. However, validity of this FFQ has been confirmed by comparison with a $3 \mathrm{~d}$ diet record, and this FFQ appears to be applicable to categorise individuals according to the intakes of energy and most nutrients ${ }^{(12)}$. Third, circulating 25-hydroxyvitamin D concentrations reflecting actual systemic vitamin D status in these individuals were not determined. Finally, as all subjects were Japanese men, the results of the present study may not be applicable to women or other ethnic populations.

In conclusion, the results of the present study demonstrate that dietary $\mathrm{Ca}$ intake is inversely associated with arterial stiffness independent of the classical atherosclerotic risk factors in Japanese men. This inverse association was striking in people with higher dietary vitamin D intake. Adequate dietary Ca and vitamin $\mathrm{D}$ intakes may act jointly to protect against the development of arterial stiffness, which eventually can lead to atherosclerosis and life-threatening cardiovascular events. Further larger and prospective or interventional studies including women and determinations of serum 25-hydroxyvitamin D concentrations that reflect systemic vitamin D status are required to confirm these findings.

\section{Acknowledgements}

The authors thank the following researchers for providing the FFQ and program to calculate nutrient intake: Shinkan Tokudome from the National Institute of Health and Nutrition (formerly Nagoya City University); Chiho Goto from Nagoya Bunri University; Nahomi Imaeda from Nagoya Women's University; Yuko Tokudome from the Nagoya University of Arts and Sciences; Masato Ikeda from the University of Occupational and Environmental Health; Shinzo Maki from the Aichi Prefectural Dietetic Association.

The present study was supported in part by Grants-in-Aid for Scientific Research on Priority Areas of Cancer (no. 17015018) and on Innovative Areas (no. 221S0001) from the Japanese Ministry of Education, Culture, Sports, Science and Technology.

The authors' contributions are as follows: H. U. and K. A. designed the research; H. U., S. K.-K., M. Y., M. N., M. H. and K. A. collected the data; H. U. and K. A. analysed the data; H. U. wrote the article; K. A. reviewed the article. All authors read and approved the final manuscript.

None of the authors has any conflicts of interest to declare.

\section{References}

1. Peterlik M \& Cross HS (2005) Vitamin D and calcium deficits predispose for multiple chronic diseases. Eur J Clin Invest 35, 290-304.

2. Umesawa M, Iso H, Ishihara J, et al. (2008) Dietary calcium intake and risks of stroke, its subtypes, and coronary heart disease in Japanese: the JPHC Study Cohort I. Stroke 39, 2449-2456.

3. Kaluza J, Orsini N, Levitan EB, et al. (2010) Dietary calcium and magnesium intake and mortality: a prospective study of men. Am J Epidemiol 171, 801-807.

4. Anderson JL, May HT, Horne BD, et al. (2010) Relation of vitamin $\mathrm{D}$ deficiency to cardiovascular risk factors, disease status, and incident events in a general healthcare population. Am J Cardiol 106, 963-968.

5. Bolland MJ, Avenell A, Baron JA, et al. (2010) Effect of calcium supplements on risk of myocardial infarction and cardiovascular events: meta-analysis. BMJ 341, c3691.

6. Lehmann ED (1999) Clinical value of aortic pulse-wave velocity measurement. Lancet 354, 528-529.

7. Tomiyama H, Yamashina A, Arai T, et al. (2003) Influences of age and gender on results of noninvasive brachial-ankle pulse wave velocity measurement - a survey of 12517 subjects. Atherosclerosis 166, 303-309.

8. Yamashina A, Tomiyama H, Takeda K, et al. (2002) Validity, reproducibility, and clinical significance of noninvasive brachial-ankle pulse wave velocity measurement. Hypertens Res 25, 359-364.

9. Hamajima N \& J-MICC Study Group (2007) The Japan Multi-Institutional Collaborative Cohort Study (J-MICC Study) to detect gene-environment interactions for cancer. Asian Pac J Cancer Prev 8, 317-323.

10. Craig CL, Marshall AL, Sjöström M, et al. (2003) International physical activity questionnaire: 12-country reliability and validity. Med Sci Sports Exerc 35, 1381-1395.

11. Tokudome S, Goto C, Imaeda N, et al. (2004) Development of a data-based short food frequency questionnaire for assessing nutrient intake by middle-aged Japanese. Asian Pac J Cancer Prev 5, 40-43.

12. Tokudome Y, Goto C, Imaeda N, et al. (2005) Relative validity of a short food frequency questionnaire for assessing nutrient intake versus three-day weighed diet records in middle-aged Japanese. J Epidemiol 15, 135-145.

13. Goto C, Tokudome $\mathrm{Y}$, Imaeda $\mathrm{N}$, et al. (2006) Validation study of fatty acid consumption assessed with a short food frequency questionnaire against plasma concentration in middle-aged Japanese people. Scand J Nutr 50, 77-82. 
14. Imaeda N, Goto C, Tokudome Y, et al. (2007) Reproducibility of a short food frequency questionnaire for Japanese general population. J Epidemiol 17, 100-107.

15. Vaskonen $\mathrm{T}$ (2003) Dietary minerals and modification of cardiovascular risk factors. J Nutr Biochem 14, 492-506.

16. Ministry of Health, Labour and Welfare, Japan (2011) The National Health and Nutrition Survey in Japan, 2011. Tokyo: Ministry of Health and Welfare (in Japanese). http://www.mhlw.go. jp/bunya/kenkou/eiyou/dl/h23-houkoku.html (accessed December 2013).

17. Ministry of Health, Labour and Welfare, Japan (Scientific Committee of Dietary Reference Intakes for Japanese) (2010) Dietary Reference Intakes for Japanese (2010 revision). Tokyo: Ministry of Health and Welfare (in Japanese). http://www.mhlw.go.jp/bunya/kenkou/kenkou_ eiyou_chousa.html (accessed December 2013).

18. Ervin RB, Wang CY, Wright JD, et al. (2004) Dietary intake of selected minerals for the United States population: 1999-2000. Adv Data 341, 1-5.

19. Kesse-Guyot E, Vergnaud AC, Fezeu L, et al. (2010) Associations between dietary patterns and arterial stiffness, carotid artery intima-media thickness and atherosclerosis. Eur J Cardiovasc Prev Rehabil 17, 718-724.

20. Iso H, Terao A, Kitamura A, et al. (1991) Calcium intake and blood pressure in seven Japanese populations. Am J Epidemiol 133, 776-783.

21. Bucher HC, Cook RJ, Guyatt GH, et al. (1996) Effects of dietary calcium supplementation on blood pressure: a meta-analysis of randomized controlled trials. JAMA 275, 1016-1022.

22. Allender PS, Cutler JA, Follmann D, et al. (1996) Dietary calcium and blood pressure: a meta-analysis of randomized clinical trials. Ann Intern Med 124, 825-831.

23. Zemel MB (2001) Calcium modulation of hypertension and obesity: mechanisms and implications. J Am Coll Nutr 20, 428S-435S, discussion 440S-442S.

24. Ledoux J, Werner ME, Brayden JE, et al. (2006) Calciumactivated potassium channels and the regulation of vascular tone. Physiology (Bethesda) 21, 69-78.
25. Resnick LM, Laragh JH, Sealey JE, et al. (1983) Divalent cations in essential hypertension. Relations between serum ionized calcium, magnesium, and plasma renin activity. $N$ Engl J Med 309, 888-891.

26. Maillard MP, Tedjani A, Perregaux C, et al. (2009) Calciumsensing receptors modulate renin release in vivo and in vitro in the rat. J Hypertens 27, 1980-1987.

27. Renaud S, Morazain R, Godsey F, et al. (1986) Nutrients, platelet function and composition in nine groups of French and British farmers. Atherosclerosis 60, 37-48.

28. Vaskonen T, Mervaala E, Sumuvuori V, et al. (2002) Effects of calcium and plant sterols on serum lipids in obese Zucker rats on a low-fat diet. Br J Nutr 87, 239-245.

29. van Meijl LE, Vrolix R \& Mensink RP (2008) Dairy product consumption and the metabolic syndrome. Nutr Res Rev 21, $148-157$.

30. Lorenzen JK, Nielsen S, Holst JJ, et al. (2007) Effect of dairy calcium or supplementary calcium intake on postprandial fat metabolism, appetite, and subsequent energy intake. Am J Clin Nutr 85, 678-687.

31. Dong Y, Stallmann-Jorgensen IS, Pollock NK, et al. (2010) A 16-week randomized clinical trial of 2000 international units daily vitamin $\mathrm{D}_{3}$ supplementation in black youth: 25-hydroxyvitamin D, adiposity, and arterial stiffness. J Clin Endocrinol Metab 95, 4584-4591.

32. Gepner AD, Ramamurthy R, Krueger DC, et al. (2012) A prospective randomized controlled trial of the effects of vitamin D supplementation on cardiovascular disease risk. PLOS ONE 7, e36617.

33. Yu A, Kim J, Kwon O, et al. (2013) The association between serum 25-hydroxyvitamin D concentration and consumption frequencies of vitamin D food sources in Korean adolescents. Clin Nutr Res 2, 107-114.

34. Nagai K, Shibata S, Akishita M, et al. (2013) Efficacy of combined use of three non-invasive atherosclerosis tests to predict vascular events in the elderly; carotid intima-media thickness, flow-mediated dilation of brachial artery and pulse wave velocity. Atherosclerosis 231, 365-370. 